\title{
Failed permacath insertion requiring cardiopulmonary bypass for resuscitation and repair
}

\author{
Galina Leyvi $^{{ }^{*}}$, Keyvan Jahanbakhsh ${ }^{2}$, Shanthan Sunku ${ }^{3}$ and Jing Song ${ }^{1}$ \\ ${ }^{1}$ Department of Anesthesiology, Montefiore Medical Center / Albert Einstein College of Medicine, USA \\ ${ }^{2}$ The Spine \& Pain Institute, Newyork, USA \\ ${ }^{3} 110$ Pine Grove Commons, Newyork, USA
}

\begin{abstract}
There are over 5 million central venous catherizations (CVCs) performed yearly in the United States [1]. Indications for CVCs include but are not limited to inaccessible peripheral venous cannulation, administration of vasoactive medications, infusion of irritating or hypertonic solutions, infusions of multiple incompatible medications, hemodialysis or hemofiltration, hemodynamic monitoring, and transvenous cardiac pacing. Studies have shown that mechanical, thrombotic, and infectious complications rates are more than $15 \%$ with CVCs [1,2]. It has been shown that the rate of mechanical complications is dependent on patient selection, site of insertion, provider experience and can range from 3.3\% [3] to up to 19\% [1] in ICU and hospital setting. Mechanical complications include failure of catheter placement, arterial puncture, improper position, pneumothorax, hematoma, and hemothorax. In recent prospective study overall rate of hemothorax was up to $0.3 \%$ [4]. In the ASA closed claims between 1970 and 2000 rate of injures related to central cathether was $1.7 \%$ (110 of 6449$)$, 15 cases of hemothorax resulted in $93 \%$ death rate [5]. As we know hemothorax is rare but often mortal complication, we report the treatment of a massive hemothorax after attempted placement of a permacath as an emergent thoracic exploration and cardiopulmonary bypass.
\end{abstract}

\section{Case History}

A 60-year-old female who was admitted for acute exacerbation of congestive heart failure (CHF) and fluid overload (Figure 1A) presented to the operating room for dialysis access via left internal jugular vein. The patient's past medical history was significant for morbidly obesity with a body mass index (BMI) of 51.5, CHF, insulin dependent diabetes mellitus, end stage renal disease requiring hemodialysis, hypertension and gastroesophageal reflux disease. The patient's only past surgical history was a left arm AV fistula. At the time of presentation the AV fistula was still patent but poorly functioning. The patient was brought to the operative suite in mild to moderate respiratory distress with oxygen via nasal cannula at $2 \mathrm{~L} / \mathrm{min}$.

She had a right sided internal jugular (IJ) triple lumen catheter as an intravenous access. The oxygen saturation $\left(\mathrm{SaO}_{2}\right)$ levels were of $95 \%-98 \%$ with the nasal cannula, otherwise her vital signs were stable. Auscultation of the lungs revealed distant breath sounds and bilateral rales. There were no other significant findings on the physical examination. An airway examination showed a short neck length, redundant neck tissue, a full range of cervical motion, Mallampati III classification, and a thyromental distance of $>6 \mathrm{~cm}$. The case proceeds with monitored anesthesia care (MAC) as $2 \mathrm{mg}$ of midazolam, $25 \mathrm{mcg}$ of fentanyl and local anesthesia at the incision site. A SonoSite ultrasound was used for the visualization and identification of the left IJ vein. A single wall needle was placed into the left IJ vein under ultrasound guidance. A Bentson guide wire was then inserted through the single wall needle and into the right atrium under view of fluoroscopic giuidance. Multiple unsuccessful attempts were made to pass a dilator using the Seldinger's technique resulting in the Bentson wire buckling. A glide catheter was placed, and the bowed wire removed. A Magic Torque wire was then inserted, and the glide catheter removed. Again, multiple unsuccessful attempts were made to pass serial dilators. Finally, an Arrow hemostatic dilator and sheath were placed and fluoroscopically visualized in the innominate vein. Several failed attempts at passing different sized and lumened catheters across the mediastium were made. At this time the surgeon attempted aspirating from the left sided catheter unsuccessfully. A venogram was performed through the catheter showing intramural contrast. The decision to abort the procedure was made, the catheter was withdrawn, and manual compression held at the site of incision. Immediately after that the patient complained of sudden onset back and chest pain, in addition to respiratory distress. She became tachycardic, hypotensive and hypoxemic. Mask ventilation had to be initiated and vasoactive medication given. The decision to obtain an intra-operative portable CXR was made (Figure 1B) to rule out a pneumothorax. No evidence of pneumothorax was demonstrated on the intraoperative CXR, however a new onset right sided layering effusion was noted. Cardiothoracic surgery was consulted, and the patient was emergently transferred to radiology for a chest CT scan (Figure 2).

The CT chest was highly suggestive of a vascular injury. Active extravasation and exact site of injury could not be determined in the non-contrast CT.

The patient continued to desaturate, became hypotensive and bradycardic. She was urgently taken to an operating room (OR) for

Correspondence to: Galina Leyvi, Department of Anesthesiology, Montefiore Medical Center / Albert Einstein College of Medicine,111 E 210th Street, Bronx, NY, USA, 10467, Tel: (917)-468-4492; E-mail: gleyvi@montefiore.org

Received: March 15, 2018; Accepted: March 26, 2018; Published: March 29, 2018 


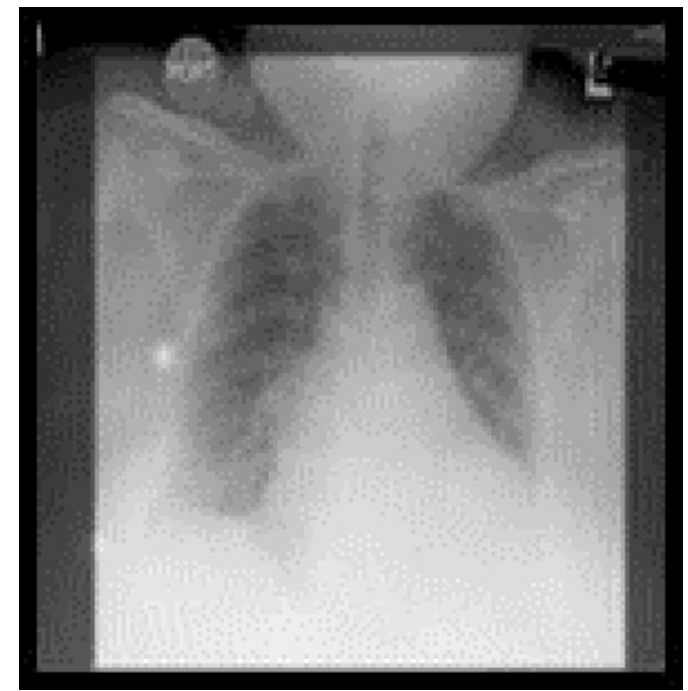

Figure 1A. Preoperative chest $x$-ray showing pulmonary vascular congestion pattern and placement of right sided central line overlying the right internal jugular vein and superior vena cava.

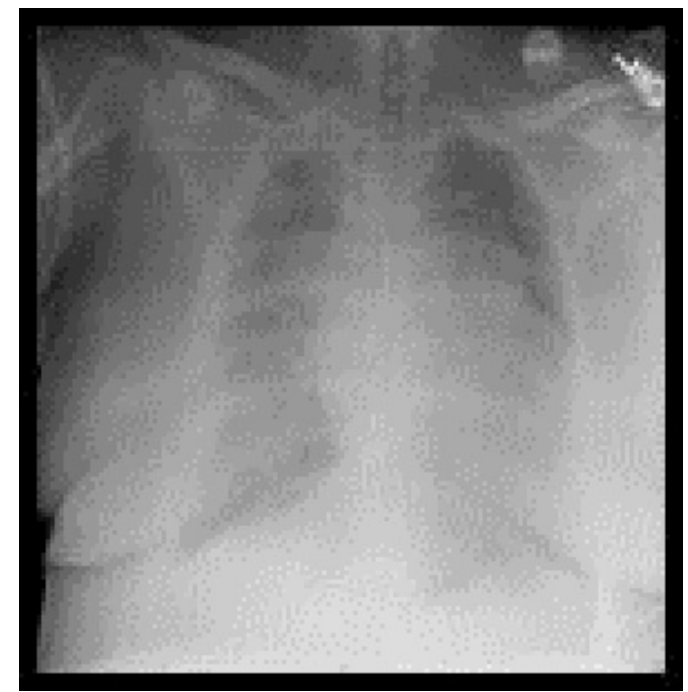

Figure 1B. Intraoperative chest $\mathrm{x}$-ray showing increased bilateral lung markings, central line with tip at the junction between the super vena cava and right atrium and layering right sided effusion.

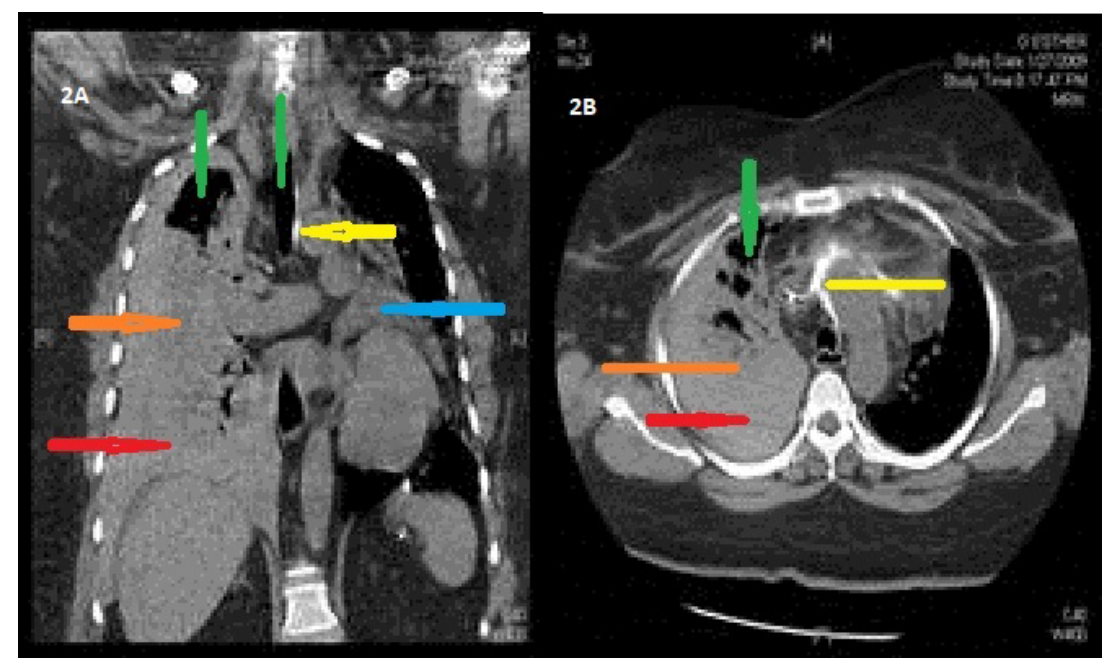

Figure 2A and 2B. CT chest without contrast showing moderate to large right hemothorax (orange), small to moderate hemopericardium (blue), mediastinal and right superior pleural air (green), extravasated contrast (from prior injection during attempted catheterization) in the mediastinum and anterior to the ascending aorta (yellow), complete collapse of right lower lobe and partial atelectasis of the right middle and upper lobes (red). 
thoracic exploration. Upon arrival to the OR cardio-pulmonary resuscitation had to be performed. The patient was difficult mask ventilation, with her $\mathrm{SaO}_{2}$ ranging between $50 \%-85 \%$ despite two hand bag valve mask ventilation and $100 \% \mathrm{FiO}_{2}$. Direct laryngoscopy (DL) and endotrachial tube (ETT) placement were unsuccessful and a laryngeal mask airway (LMA) was placed. Poor gas exchange and oxygenation continued with the LMA and renewed attempts to place an ETT were made using a fiberoptic scope, Glide Scope and fast-track LMA. Eventually, an ETT was placed via DL by a third attending anesthesiologist. Following ETT placement poor ventilation continued with $\mathrm{SaO}_{2}$ ranging between $70 \%$ to $85 \%$, increased peak inspiratory pressures $>40 \mathrm{cmH}_{2} \mathrm{O}$ and systolic blood pressure in 70 s. Transesophageal echocardiogram revealed poor right ventricle function and an empty left ventricle.

An emergency median sternotomy was performed, with the right and left pleura opened. Approximately $700 \mathrm{ml}$ of blood and clot was evacuated from the right chest. A small amount of serous fluid was found within the pericardium along with blood infused pericardial fat. After chest decompression ventilation, oxygenation, peak pressures and systemic blood pressure considerably improved. An actively exsanguinating laceration of the innominate vein overlying the aortic arch was discovered. The patient had to be placed on cardiopulmonary bypass to ligate the innominate vein. The patient was stabilized and subsequently transferred to the cardiac intensive care unit for further care.

\section{Discussion}

In our literature search, we could not find any reported cases of hemothorax after CVC insertion requiring cardiopulmonary bypass as a primary resuscitation intervention and method for repair.

Our case report is an example of a hemothorax as a mechanical complication of CVCs and specific patient risk factors. Two prospective studies found the rates of all mechanical complications to be $3.3 \% 3$ and $14 \%$ 4. Risk factors associated with mechanical CVC complications include high and low BMI, prior CVCs and surgeries, prior radiotherapy, advanced age, number of percuntaneous punctures, insertion site, gender, number of percutaneous punctures, and time needed for catheter placement $[4,6]$. While studies have shown that limiting the number of percutaneous punctures substantially reduces the risk of mechanical complications 4,6 , our patient only had a single percutaneous puncture by an experienced provider with ultrasound guidance

It is noteworthy to mention the use of dilators by the surgical team. Oropello, et al. [7] reported two cases of double-lumen hemodialysis catheter insertion (one internal jugular vein placement and another inadvertent arterial placement) were it was believed that dilator associated complications resulted in fatal hemothorax. Due to the length and stiffness of the dilators, forceful insertion may cause the dilator to not follow the flexible wire as a guide and cause great vessel or cardiac chamber perforation [7]. Both patients suffered cardiac arrest shortly after insertion. Our patient showed more tolerability holding for 2 hours during CT scan evaluation, and immediate availability of cardiac surgeon was life saving.

As previously mentioned the incidence of hemothorax because of CVCs is low. However, the mortality associated with the complication is higher than with all other CVC complications combined 5. A delay in hemothorax detection can be a serious and deadly complication. While there are many methods for detection of misplaced catheters and their complications they are not infallible. Common techniques used for verification of correct intravascular placement include aspiration of blood and frontal CXR. It has also become widespread to use real time ultrasonography guidance for CVC placement $[8,9]$. Yet, even with the use of all these methods absolute confirmation of correct catheter placement cannot be made as exemplified by our case report.

Our patient's AV fistula may have contributed to the severity and rapidity of her hemothorax development by arterialization of her innominate vein. As noted by the cardiothoracic surgeons there was active hemorrhaging from the injury site. Similarly, Collini, et al. [10] report a case where it was believed that the presence of an AV fistula contributed to hemothorax formation because of ipsilateral CVC removal. They recommend CVCs on the same side as an AV fistula to be avoided. Moreover, common methods for treating the patient's hemothorax, i.e. a chest tube, would likely have not worked in this situation; only once the patient was placed on cardiopulmonary bypass could an attempt to repair the injury be made due to the active bleeding from the laceration.

It is our recommendation that further studies should be performed on the complication rates associated with the use of dilators during placement of CVC and CVCs complications in patients with AV fistulas and grafts.

\section{References}

1. McGee DC, Gould MK (2003) Preventing complications of central venous catheterization. The New England Journal of Medicine. 348: 1123-1133. [Crossref]

2. Taylor RW, Palagiri AV (2007) Central venous catheterization. Critical Care Medicine 35: 1390-1396. [Crossref]

3. Schummer W, Schummer C, Rose N (2007) Mechanical complications and malpositions of central venous cannulations by experienced operators. A prospective study of 1794 catheterization in critically ill patients. Intensive Care Medicine 33: 1055-1059. [Crossref]

4. Eisen LA, Narasimhan M, Berger JS (2006) Mechanical complications of central venous catheters. Journal of Intensive Care Medicine 21: 40-46. [Crossref]

5. Domino KB, Bowdle TA, Posner KL (2004) Injuries and liability related to central vascular catheters: a closed claims analysis. Anesthesiology 100: 1411-1418. [Crossref]

6. Takeyama H, Taniguchi M, Sawai H (2006) Limiting vein puncture to three needle passes in subclavian vein catheterization by the infraclavicular approach. Surgery today 36: 779-782. [Crossref]

7. Oropello JM, Leibowitz AB, Manasia A (1996) Dilator-associated complications of central vein catheter insertion: possible mechanisms of injury and suggestions for prevention. Journal of Cardiothoracic and Vascular Anesthesia. 10: 634-637. [Crossref]

8. Wiklund CU, Romand JA, Suter PM (2005) Misplacement of central vein catheters in patients with hemothorax: a new approach to resolve the problem. The Journal of Trauma 59: 1029-1031. [Crossref]

9. Deogaonkar K, Shokrollahi K, Dickson WA (2007) Haemothorax: A potentially fata complication of subclavian cannulation-A case report. Resuscitation 72: 161-163. [Crossref]

10. Collini A, Nepi S, Ruggieri G (2002) Massive hemothorax after removal of subclavian vein catheter: a very unusual complication. Critical care medicine 30: 697-698. [Crossref]

Copyright: (C2018 Leyvi G. This is an open-access article distributed under the terms of the Creative Commons Attribution License, which permits unrestricted use, distribution, and reproduction in any medium, provided the original author and source are credited. 